\title{
Selected converter topologies for interfacing energy storages with power grid
}

\author{
A. DOMINO, K. ZYMMER*, and M. PARCHOMIUK
}

Electrotechnical Institute, 28 Pożaryskiego St., 04-703 Warsaw, Poland

\begin{abstract}
The paper presents different solutions applicable in power converter systems for connecting power grids with energy storage systems such as superconducting magnetic energy storage (SMES), supercapacitor energy storage (SES) or chemical batteries. Those systems are characterized by bidirectional current flow between energy storage and power grid. Two-level converters (AC-DC and DC-AC converters) dedicated for low power energy storage compatible with $3 \times 400 \mathrm{~V}$-type power grids are proposed. High power systems are connected with $3 \times 6 \mathrm{kV}$-type power grids via transformers that adjust voltage to the particular energy storage or directly, based on multilevel power converters (AC-DC and DC-AC) or dual active bridge (DAB) systems. Solutions ensuring power grid compatibility with several energy storage systems of the same electrical parameters as well as of different voltage-current characteristics are also proposed. Selected simulation results illustrating operation of two system topologies of $200 \mathrm{~kW}$ power for two-level converter and neutral point clamped (NPC) three-level converter are presented.
\end{abstract}

Key words: power converter, superconducting magnetic energy storage (SMES), supercapacitor energy storage (SES), distribution system.

\section{Introduction}

Recently, due to the fast development of renewable energy sources (RES), smart grids and ultra-fast charging stations (UFCS), the interest in installing different types of energy storages has considerably grown [1-3]. Selected storage technologies, their descriptions and specifications are presented in [4-8]. Power electronic systems are designed to operate with different energy storage systems, such as super-magnetic energy storage (SMES), batteries (accumulators) or supercapacitors (SES). Basically, the power electronic grid interface should have the following features $[9,11,13,15]$ :

- bi-directional energy flow in both parts of the AC and DC converter sides,

- the possibility of adjusting the parameters of the electricity supplied or taken from the energy storage.

This paper presents a review of selected power converter topologies which can be used as a coupling between the power grid and energy storage [9]. The systems discussed in this paper are mainly designed to maintain power supply of industrial installations in case of power supply failure for at least the time period required for the controlled shutdown or end of the process - at least for a minute or even longer [3]. This is important in cases when the sudden interruption in the process makes it necessary to revise the troublesome installation, for example the output of plastic products, powered electrical furnaces for melting metals, rolling mills, electrochemical processes, etc. Such systems may also be utilized for storing energy at a time when the cost is low (night-time tariff), and using it at a time when the price increases significantly. Another important feature is the possi-

*e-mail: k.zymmer@iel.waw.pl

Manuscript submitted 2017-05-11, revised 2017-09-01 and 2017-09-08, initially accepted for publication 2017-09-08, published in October 2017. bility of limiting the temporary increases in power drawn from the grid during start-up of high-power equipment and operation under overload. Note that the hybrid group of energy storage systems with different current-time characteristics, described in this paper, can assure simultaneous compensation of the peak power and long overload state.

The peak power is an essential parameter of the electricity tariff in industrial plants. The systems coupling power grid with energy storage can be divided into two groups:

- voltage systems referred to the energy storages as supercapacitors (SES) and battery trays [17, 20-27],

- current systems for SMES energy storage type [28-34],

In those solutions an active rectifier is connected to the grid [28], which results from the requirement of bi-directional energy flow of an energy storage, operating directly with bi-directional DC-DC converter [14]. This solution provides both powering and returning energy to the grid with unity power factor. The paper discusses selected topologies of converter systems connecting the energy storage and power grid with different rating parameters using transformers and transformer-less solutions.

\section{Converter topologies for interfacing energy storages with grid}

2.1. Topology 1. The basic scheme of the arrangement is shown in Fig. 1, where superconducting choke is used as the energy storage element - SMES. The system consists of an AC-DC converter connected to the power grid and the bi-directional DC-DC converter in the form of the pulse current regulator [13]. It is also possible to use battery storage or supercapacitors cooperating with the DC-DC pulse voltage regulator. Electrical parameters of the converter such as current, voltage and power characteristics are determined by parameters of the energy storage [15]. 
Table 1

Main technical features of storage technologies

\begin{tabular}{|c|c|c|c|c|c|c|c|}
\hline Parameter/Technology & $\begin{array}{l}\text { Power } \\
{[\mathrm{MW}]}\end{array}$ & $\begin{array}{c}\text { Storage duration } \\
{[\mathrm{h}]}\end{array}$ & $\begin{array}{l}\text { Cycling } \\
\text { or lifetime }\end{array}$ & $\begin{array}{l}\text { Energy density } \\
\text { (Wh/l) }\end{array}$ & $\begin{array}{l}\text { Power density } \\
\text { (W/l) }\end{array}$ & Efficiency & $\begin{array}{l}\text { Response } \\
\text { time }\end{array}$ \\
\hline Pumped hydro storage (PHS) & $100-1000$ & $4-12 \mathrm{~h}$ & $30-60$ years & $0.2-2$ & $0.1-0.2$ & $70-85 \%$ & Sec.-Min. \\
\hline Flywheels & $0.001-1$ & Seconds-hours & $20000-100000$ & $20-80$ & 5000 & $70-95 \%$ & $<\mathrm{Sec}$ \\
\hline Sodium sulfur $(\mathrm{NaS})$ battery & $10-100$ & $1 \mathrm{~min}-8 \mathrm{~h}$ & $2500-4500$ & $150-300$ & $120-160$ & $70-90 \%$ & $<\mathrm{Sec}$ \\
\hline Li-ion battery & $0.1-20$ & $1 \mathrm{~min}-8 \mathrm{~h}$ & $1000-10000$ & $200-400$ & $1300-10000$ & $85-98 \%$ & $<\mathrm{Sec}$ \\
\hline Vanadium redox flow battery & $0.1-100$ & $10 \mathrm{~h}$ & $12000-14000$ & $20-70$ & $0.5-2$ & $60-85 \%$ & $<\mathrm{Sec}$ \\
\hline Supercapacitor (SES) & $0.01-1$ & Msec-min & $10000-100000$ & $10-20$ & $40000-120000$ & $80-98 \%$ & $<\mathrm{Sec}$ \\
\hline $\begin{array}{l}\text { Superconducting magnetic } \\
\text { energy storage (SMES) }\end{array}$ & $0.1-1$ & Msec-sec & 100000 & $\sim 6$ & $\sim 2600$ & $80-95 \%$ & $<\mathrm{Sec}$ \\
\hline Hydrogen & $0.01-1000$ & Minutes-weeks & $5-30$ years & 600 & $0.2-20$ & $25-45 \%$ & Sec-Min \\
\hline
\end{tabular}

Depending on the relationship between the grid voltage and the nominal voltage of the energy storage, the converter system may operate as transformer-less or be equipped with a grid-transformer. Control parameters of the transmitted energy for both charging and discharging of the energy storage is implemented in a bi-directional DC-DC pulse current controller between the switched capacitor CF in DC-link and SMES energy storage inductor. In this solution, it is desirable to use the unipolar control, because the current in the circuit between the capacitor and the energy storage is much lower than in the case of classic bipolar control $[13,15]$. This has an impact on the selection of capacitors in the DC-link, as well as the price of those elements. Active rectifier in the form of a vector-controlled three-phase two-level transistor bridge provides high system efficiency, optimum currents at active power flow between the grid and the rectifier, as well as operation with unity power factor $[12,16]$. The LC filter at the input of the rectifier reduces the emission of harmonics in voltage and current transferred from the energy storage to the grid. In the case of SMES storage, the topology allows for connecting several inductors in the storage circuit. Those inductors should have similar inductance values and current ratings. The discussed topology is simple and contains a minimum number of components and measuring transducers. It is addressed to small and medium power systems supplied with $3 \times 400 \mathrm{~V}$ grids operating without grid-transformer. In the presented topology, a two-level active rectifier from the grid side and two-level DC-DC controller from the energy storage side is applied. It is the cheapest solution, but it has drawbacks such as the deformation of the voltage and grid current waveforms. However, they can be largely compensated by using a suitable LC filter from the grid side.

2.2. Topology 2. The topology shown in Fig. 2, is a modification of the variant described above. It assumes bi-directional energy flow with rated power higher than the system of Fig. 1. This is achieved thanks to the use of medium voltage grid-transformer with a significantly higher short-circuit power than for a $3 \times 400 \mathrm{~V}$ grid. The energy conversion system can work with several storages with different power capacity, but with a similar level of operating voltage. The discussed topology may include some type of energy storage SMES working in parallel and connected to bi-directional pulse current regulators. This structure may also include a SES supercapacitor or battery storage connected to the DC-link via the pulse voltage regulator. Both types of pulse controllers used here provide the required direction and the amount of energy transferred between the storage battery and capacitor $\mathrm{CF}$ in the DC-link. Active rectifier connected between grid-transformer and capacitor $\mathrm{CF}$ provides energy flow between grid or energy storage. The system with

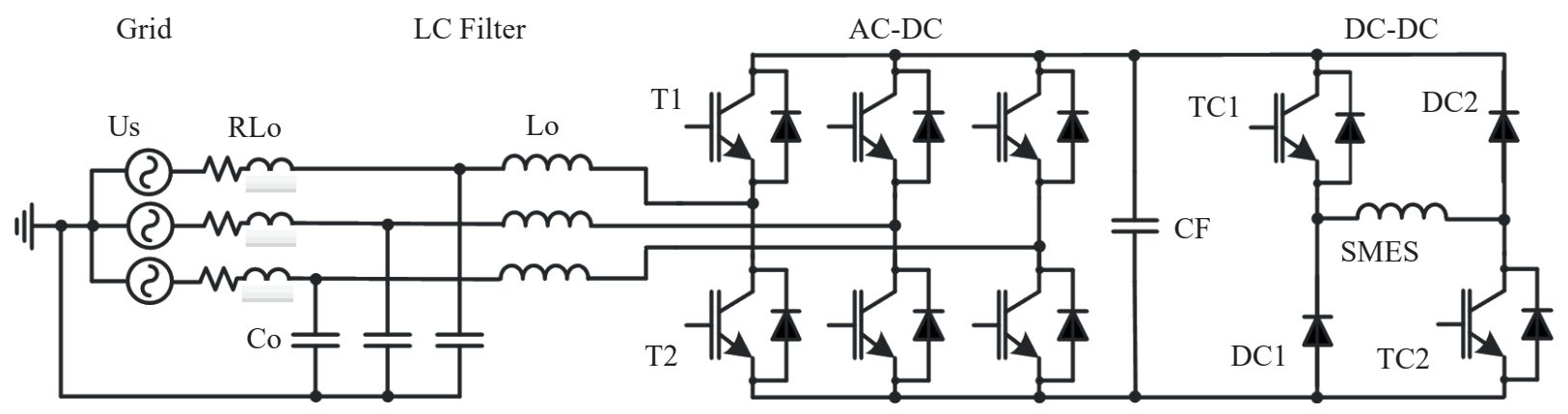

Fig. 1. Topology No.1 with a single SMES, a two-level three-phase AC-DC active rectifier and two-level DC-DC converter 
Grid Transformer LC Filter

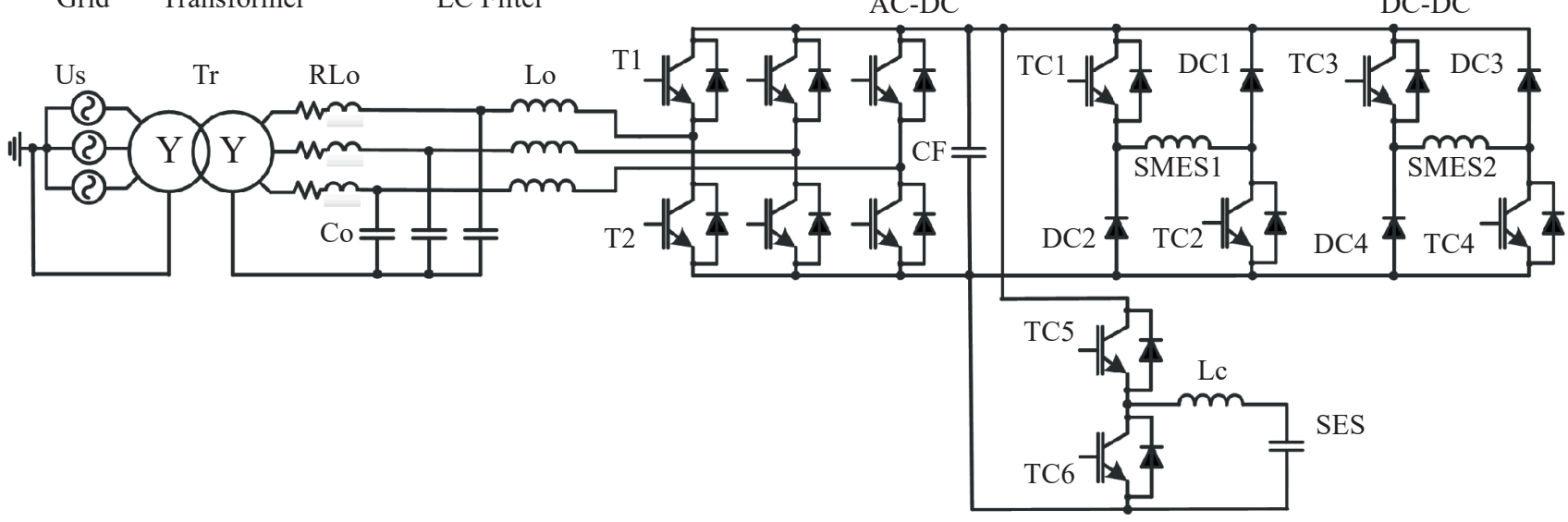

Fig. 2. Topology No. 2 with two SMES and one capacitor storage, two-level three-phase active rectifier, and three two-level DC-DC converters

Grid $\quad$ LC Filter

AC-DC

DAB

DC-DC

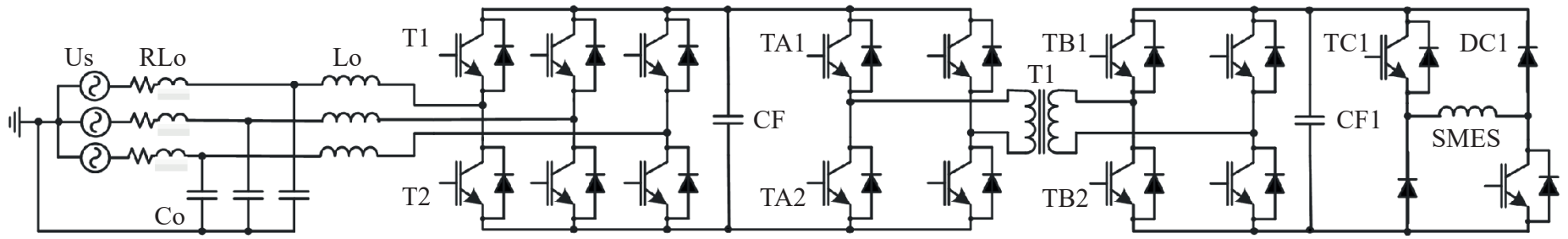

Fig. 3. Topology No. 3 with single SMES, 2-level 3-phase active rectifier, system fit with DAB converter and two-level DC-DC converter

a two-level converter has the same advantages and disadvantages as those outlined in the discussion of Topology 1.

2.3. Topology 3. This topology is aimed for low and medium power systems supplied directly from a power grid with a voltage of $3 \times 400 \mathrm{~V}$. It includes the dual active bridge (DAB) with a high frequency transformer guaranteeing low weight and dimensions $[18,19]$. This solution provides the accurate transmission system voltage, which is assessed by the grid according to the requirements of the energy storage - for example, low op- erating voltage, which can be the case with currently used small energy capacity SMES storage. In addition, the DAB transformer provides galvanic isolation between the energy storage and the grid. This topology, when compared with Topology 1, has a higher number of power electronics components, but ensures the use of low operation voltage storage. The system is based on a two-level converter.

2.4. Topology 4. This solution constitutes a development of Topologies 2 and 3 discussed above. The topology allows for

Grid Transformer $\quad$ LC Filter $\quad$ AC-DC

$\mathrm{DAB}$

DC-DC

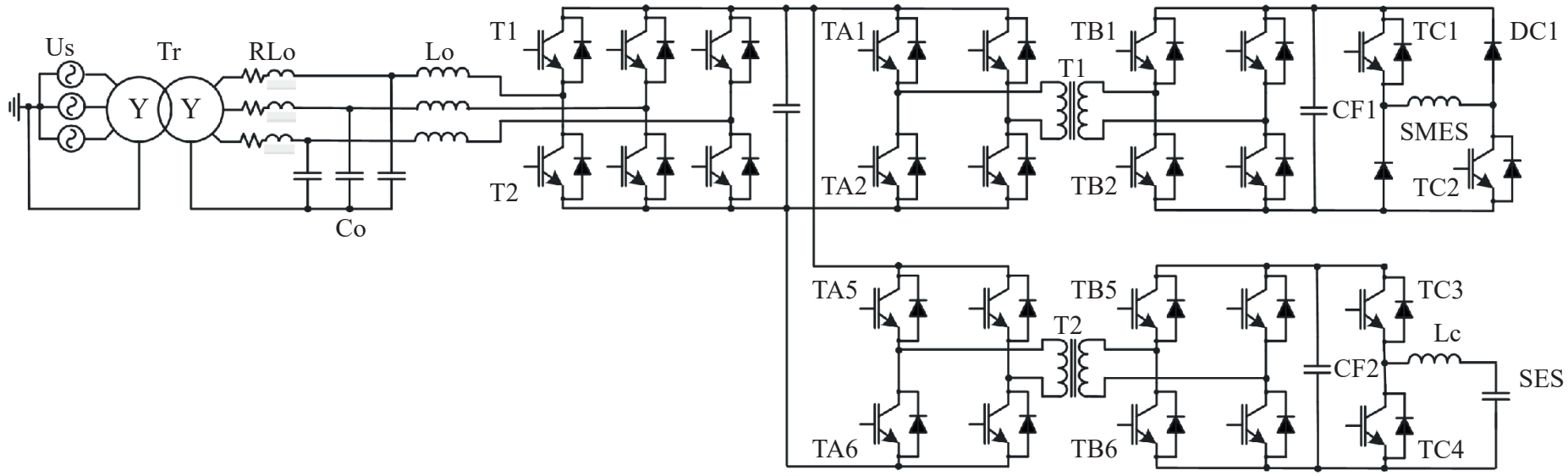

Fig. 4. Topology No. 4 with the SMES and capacitor, two-level three-phase active rectifier, two DAB converters, two DC-DC converters 


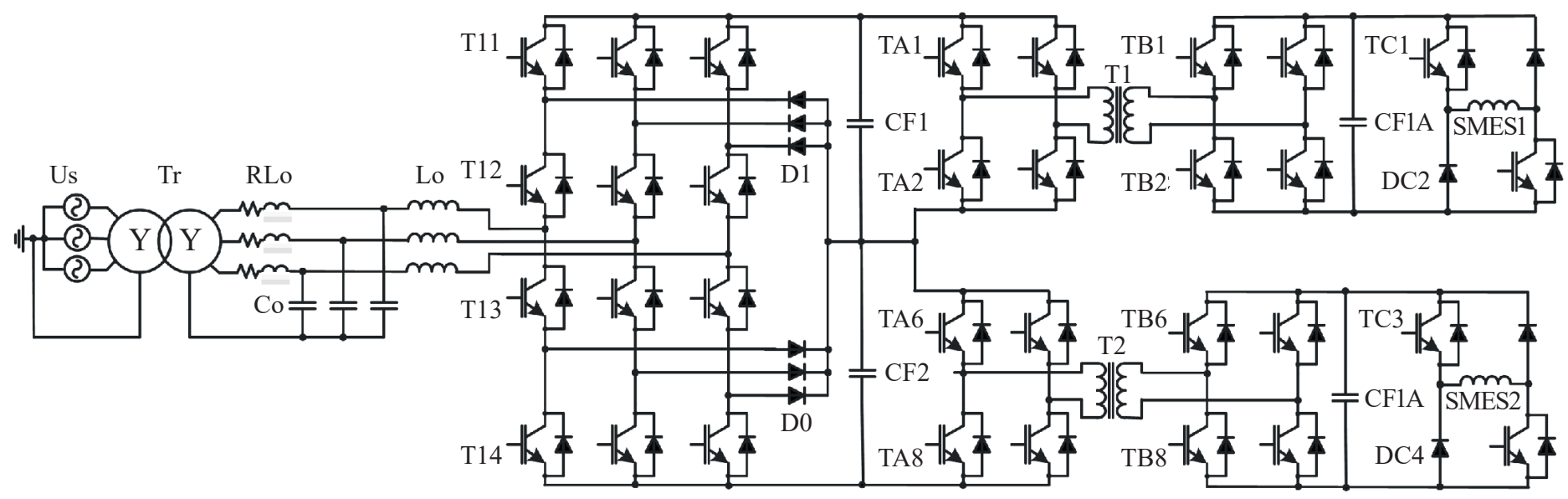

Fig. 5. Topology No. 5 with two SMES, three-level three-phase active rectifier, two matching DAB converters, and two 2-poziomowymi DC-DC converters

paralleling multiple energy storage with different performance ratings, such as the energy capacity, power and operating voltage. It is addressed to systems of medium and high power cooperating with one grid voltage and using the simultaneous operation of all kinds of energy storage.

DAB systems operating in parallel with the high-frequency transformers allow for adjustment of various operating voltages of energy storage to the required voltage in the DC-link, which is necessary for the energy transfer between the storage system and grid. When working with medium voltage grid (the energy storage systems are generally adapted to the voltage of several hundred volts), it is required to install power grid-transformer adapting power supply voltage to the requirements of energy storage.

2.5. Topology 5. In combination with the electricity grid of $6 \mathrm{kV}$ voltage, it is possible to use the direct supplied active multilevel rectifier and two-level DC-DC converters supplying the primary windings of high frequency DAB transformers. This solution is feasible but can be assessed as less favorable for reasons of safety and a higher complexity for both the main circuit and control circuit. Another drawback associated with the operation of power converters for high voltage is the reduction of switching frequency. This is due to the dynamic properties of the power electronic semiconductor devices and the generated switching power loss with increasing frequency and voltage. Reducing the switching frequency of energy conversion is a barrier to the miniaturization of transformers adapting the voltage level to energy storage requirements. It should be noted that multilevel converters are capable of reducing the impact of higher harmonics on current and voltage waveforms in the system. Moreover, using a higher voltage in the DC circuit than that required by the electric energy storage may be advantageous in high power systems due to the current limiting at predetermined power. In the arrangement shown in Fig. 5, the adopted structure of the three-level inverter as an active rectifier (with the possibility of increasing the number of levels), and two-level DAB systems cooperating with pulse regulators of DC-DC converters connected directly to the energy storage SMES1 and SMES2 are presented.

A limitation in the flexibility of the system stems from the fact that different systems work with DAB capacitor batteries CF1-CFN of equal capacity, which requires the symmetry of voltages at different levels of the DC active rectifier connected to the grid. Maintaining the same voltages on these capacitors requires both the charge and discharge of the same energy. Thus, the system is dedicated to work with a certain number of stores of the same type and with similar energy capacities.

2.6. Topology 6. The system of power transfer shown in Fig. 6 can be used with power grid $3 \times 400 \mathrm{~V}$ without grid side transformer or with grid-transformer when connected to the medium voltage grid, for example, $6 \mathrm{kV}$. The active three-level rectifier (applied at low side voltage) reduces harmonic current and voltage for bidirectional energy flow. This results in the reduction of grid side filter capacitance and inductance. The condition for the correct operation of the system, similarly as in Topology 5 , is to use the same type of energy storage capacitors cooperating with the DC-link. They should be of the same energy storage capacity, and the same power when they are loading and unloading. This structure using a converter with a larger number of levels (e.g. five-level) could co-operate directly with the network of $6 \mathrm{kV}$, using the appropriate number of identical energy storage units.

2.7. Topology 7. The structure shown in Fig. 7 co-operates with a single energy storage unit of large capacity. This solution can be used for direct cooperation with the supply grid $3 \times 400 \mathrm{~V}$ or with a grid of different voltage (using the grid side transformer). This system employs a three-level active rectifier from the grid and three-level regulator attached to the energy storage. Similarly as in Topology 6, it reduces the value of harmonic current and voltage for both directions of energy flow. The introduction of a three-level DC-DC pulse current controller cooperating 
Grid Transformer $\quad$ LC Filter

DC-DC

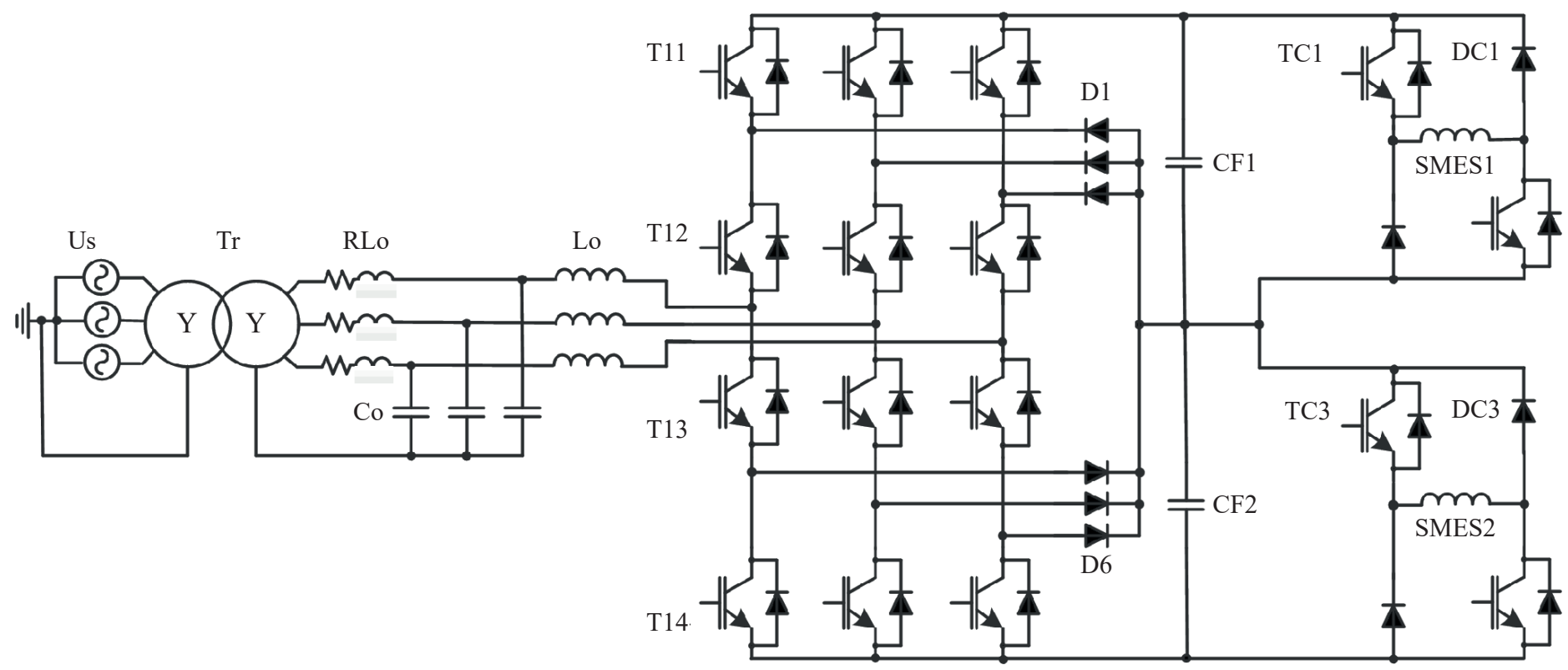

Fig. 6. Topology No. 6: with n SMES, n-level three-phase active rectifier, and DC-DC converters operating in parallel

Grid Transformer $\quad$ LC Filter

DC-DC

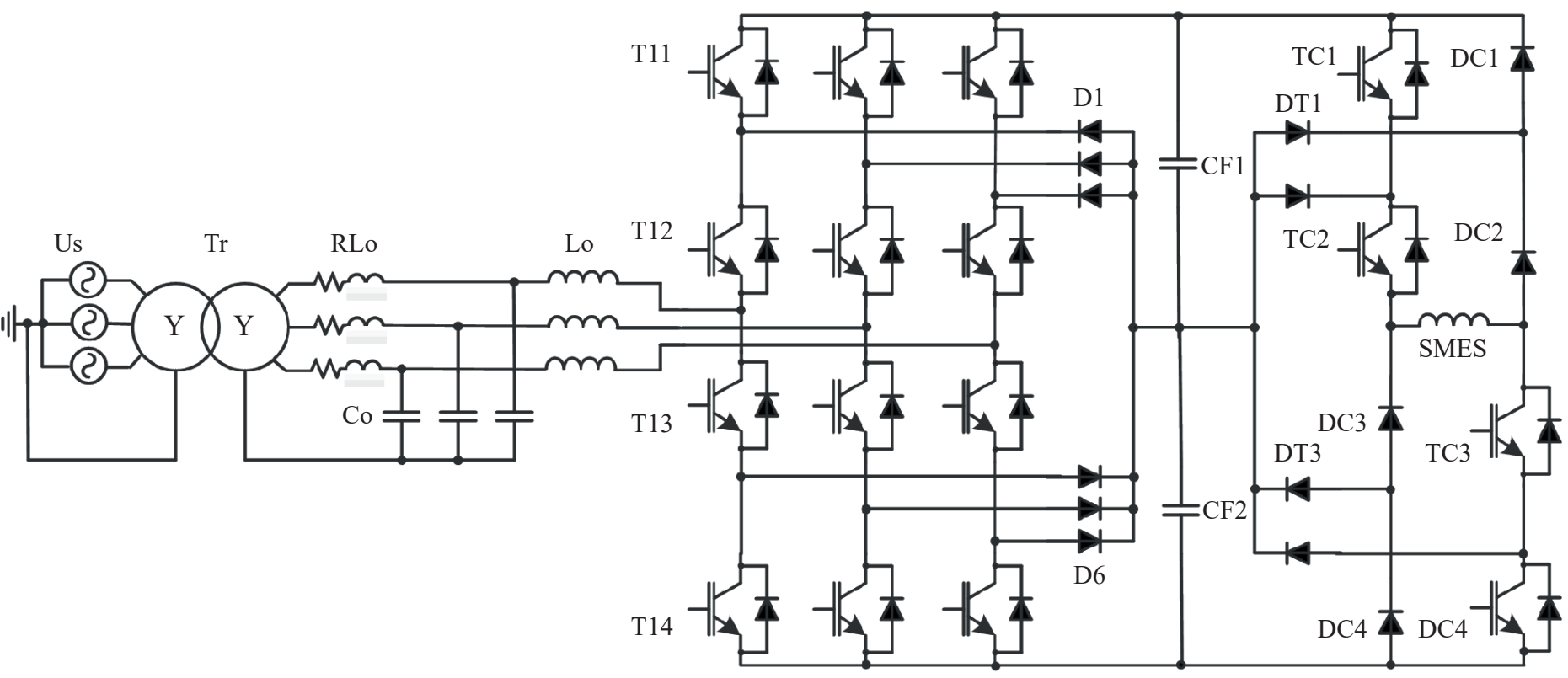

Fig. 7. Topology No. 7 with single SMES, three-level three-phase active rectifier, and three-level pulse DC-DC regulator

with the energy storage helps to achieve voltage symmetry in DC-link capacitors CF1 and CF2. In this system (Fig. 7), the use of a single energy storage unit results in doubling the current in the storage circuit configuration discussed in Topology 6 at the same voltage of DC-link capacitors. This topology, without a grid-transformer, is dedicated for energy storage with a limited current capacity available in the low voltage grid. With the power supply from the medium-voltage grid by matching transformer it is possible to convert electricity with more lim- ited power, but with permissible current conduction of semiconductor devices.

\section{Simulation results}

In order to design the power converter systems for energy storage and to illustrate operation of the selected topologies, simulation models were developed using PSIM 10.0 package 

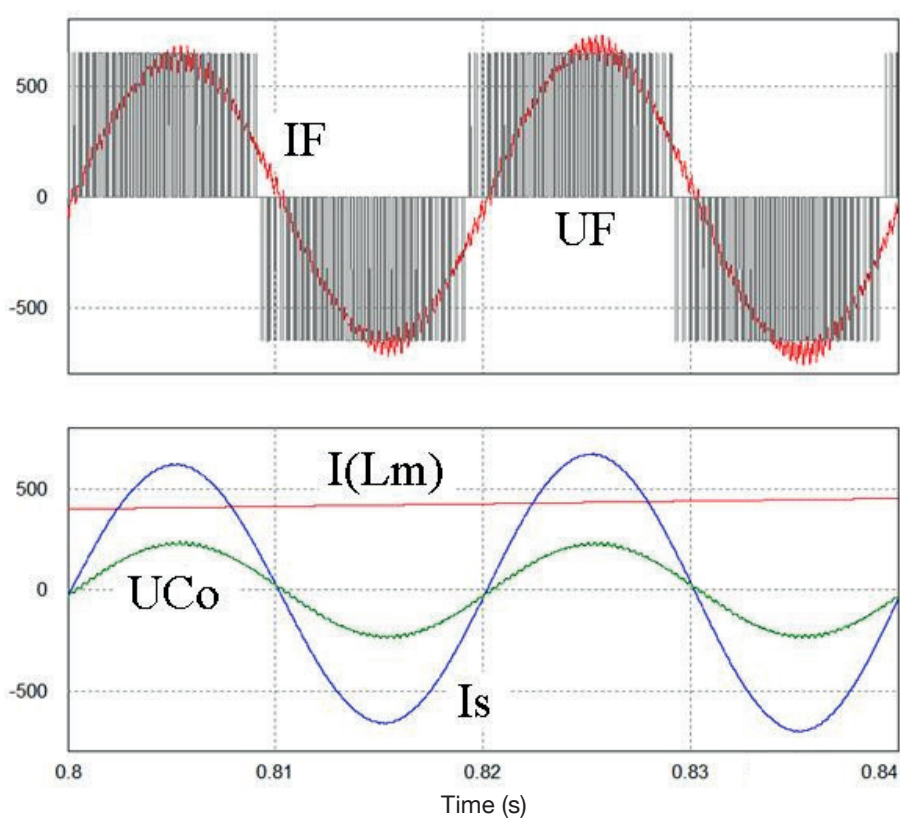

Fig. 8. Charging process of SMES in Topology 1 with two-level AC/ DC grid-side converter and the bi-directional DC-DC converter; the energy is taken from the grid; UF - phase to phase input voltage without filter, IF - grid current without input filter, I(Lm) - SMES current, Is - grid current after input filter, UCo - phase input voltage

and selected results are presented in Figs. 8-18. Oscillograms in Figs. 8-10 show operation of an interface of power converter system, in which the power block consists of an twolevel grid-connected active rectifier AC-DC the bi-directional
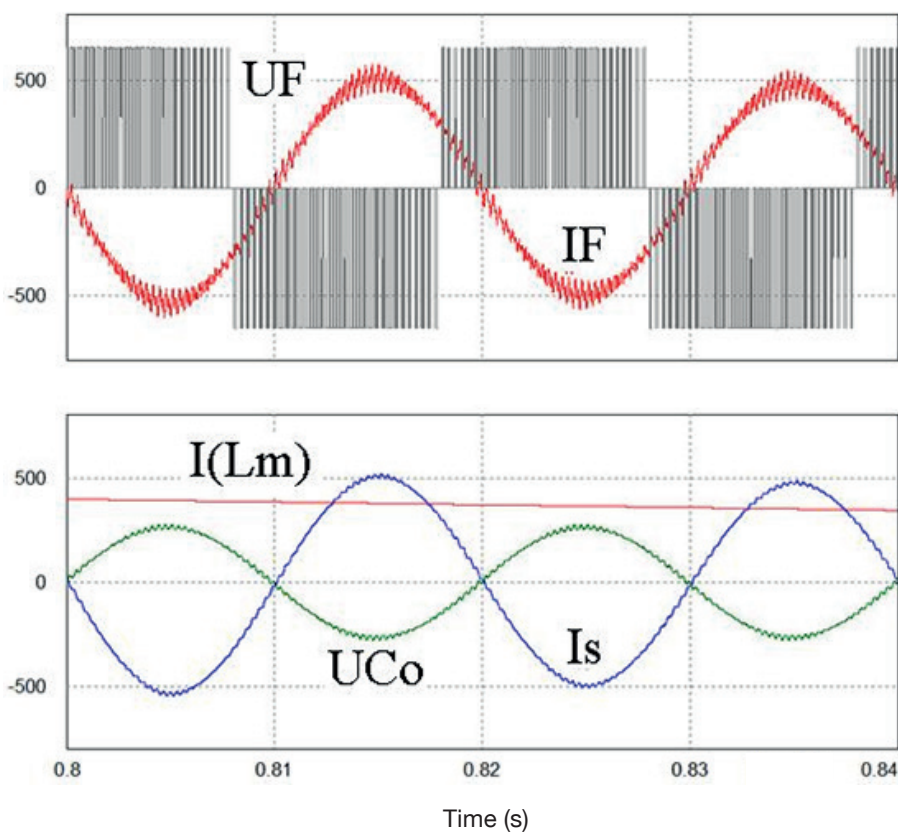

Fig. 9. Discharging process of SMES, energy is flowing back to the grid. UF - phase to phase input voltage without filter, IF - grid current without input filter, I(Lm) - SMES current, Is - grid current after input filter, $\mathrm{UCo}-$ phase input voltage
DC-DC converter in the form of the pulse current regulator, according to the topology presented in Fig. 1. As stated, this topology is simple and includes a limited number of components in the main circuit and the measurement, which means that its price is favorable. It is also characterized by high reliability. Figures 8 and 9 present charging and discharging processes with $\mathrm{I}(\mathrm{Lm})=400 \mathrm{~A}$ of output current, where electric energy is flowing to the SMES and back to the grid respectively at $215 \mathrm{~kW}$ of rated power. To show the current change $\mathrm{I}(\mathrm{Lm})$ during charging and discharging SMES, the inductance $\mathrm{Lm}=0.4 \mathrm{H}$ was taken for simulation; for large values of inductance the current changes I $(\mathrm{Lm})$ would be barely visible. The DC-link voltage is $\mathrm{Udc}=650 \mathrm{~V}$.

The modulation frequency of sinusoidal PWM of AC-DC converter is $f_{p r}=5 \mathrm{kHz}$ and operating frequency of the DC-DC converter is $f_{c z}=4 \mathrm{kHz}$. When the energy flows from the grid, the grid current and voltage are in phase, which means the energy storage system takes pure active power. During the discharging process, when energy is transferred back to the power grid, the phase current and voltage are in opposition. In this situation only active energy is transferred to the grid.

Figures 10 shows the processes during operation of the bi-directional DC-DC converter (see Fig. 1) during charging and discharging energy in SMES. It presents the instantaneous current shape of inductor IoC and the ULm voltage in the SMES inductor, against the background of the effective current IoCrms $=364 \mathrm{~A}$ and the current $\mathrm{I}(\mathrm{Lm})$ flowing into the SMES.

Figures 11 and 12 illustrate processes occurring during charging and discharging process in SMES in the topology containing the DAB circuit shown in Fig. 3. The voltage in the DC-link of the active rectifier is $\mathrm{Udc}=650 \mathrm{~V}$. The
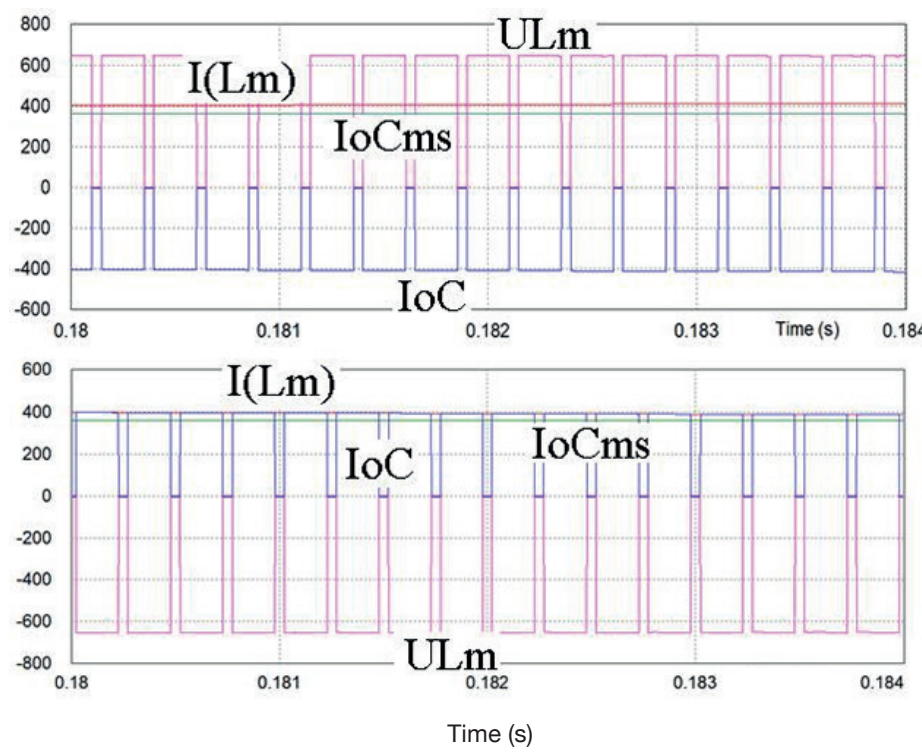

Fig. 10. Charging process (top) and discharging (bottom) of SMES in bi-directional DC-DC converter (structure from Fig. 1), IoC - instantaneous inductor charging current, $\mathrm{I}(\mathrm{Lm})$ - current flowing in SMES, ULm - voltage on SMES, IoCrms - effective value of inductor charging current 

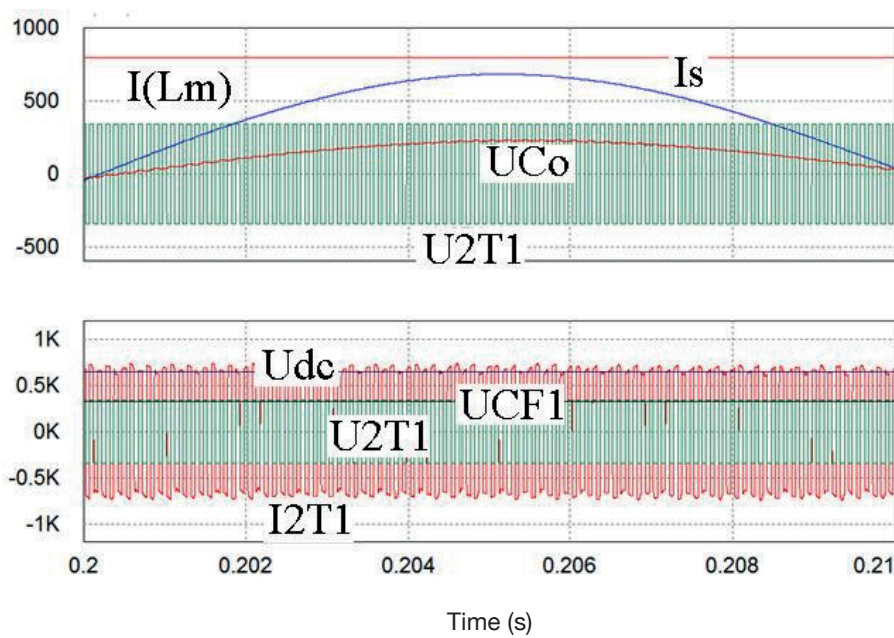

Fig. 11. Charging process of SMES in Topology 3 with DAB. I(Lm) - SMES current, Is - grid current after input filter, UCo - phase input voltage, $\mathrm{U} 2 \mathrm{~T} 1$ - secondary winding voltage of $\mathrm{T} 1$ transformer. Udc-dc link voltage, UCF1-capacitor voltage CF1, I2T1, U2T1 - secondary winding current and voltage of transformer T1

SMES voltage is UCF $1=330 \mathrm{~V}$ and the SMES current is $\mathrm{I}(\mathrm{Lm})=800 \mathrm{~A}$. Active power determined by current and phase voltage (Is, UCo) $\mathrm{P}=230 \mathrm{~kW}$. The DAB transformer secondary winding current is $\mathrm{I} 2 \mathrm{~T} 1=340 \mathrm{~A}$. Figures 11 and 12 show shapes of the U2T1 voltage and the I2T1 currents of the secondary winding of $\mathrm{DAB}$ transformer in relation to the voltage and phase current (UCo, Is) of the active rectifier and the storage current I $(\mathrm{Lm})$, the voltage in the DC-link (Udc)
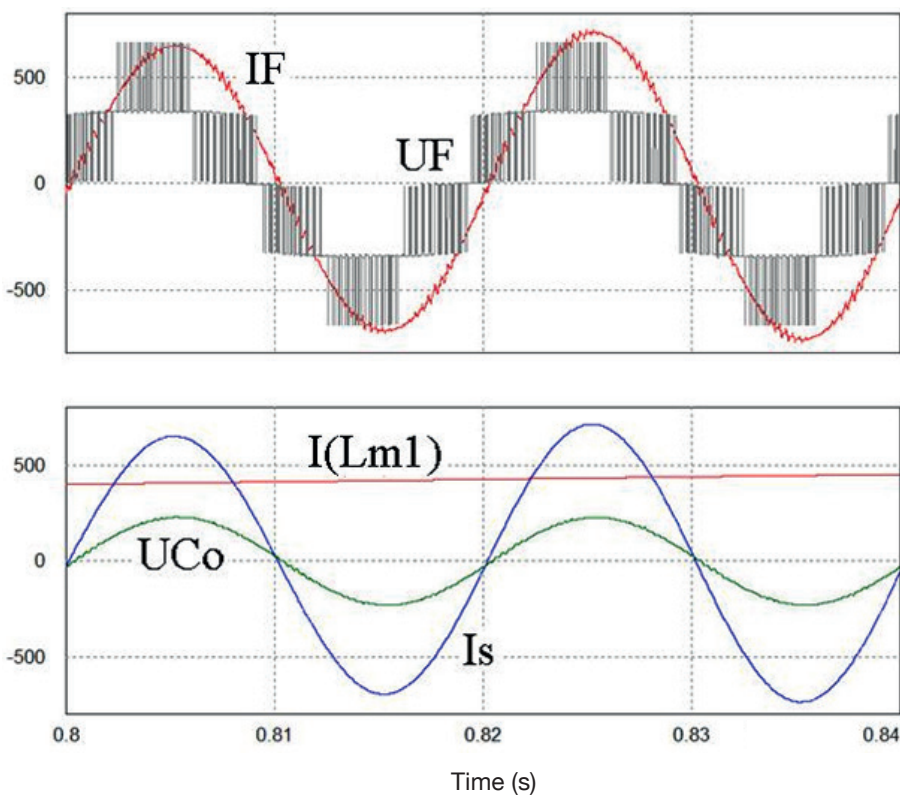

Fig. 13. Charging process of SMES in Topology 6 with threelevel AC-DC grid-converter, and two bi-directional DC-DC converters; energy is taken from the grid. UF - phase to phase input voltage without filter, IF - grid current without input filter, I(Lm) - SMES current, Is - grid current after input filter, UCo - phase input voltage
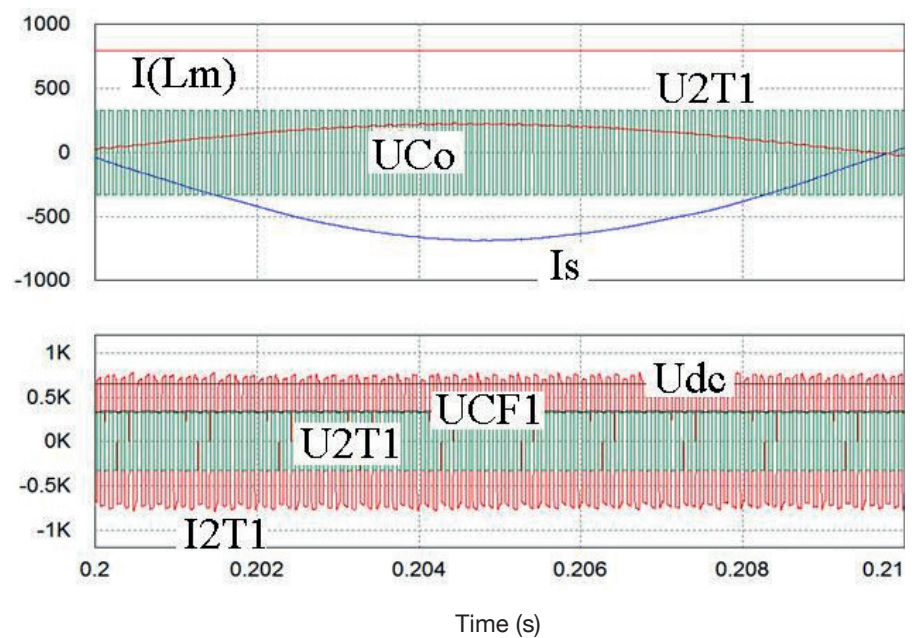

Fig.12 Discharging process of SMES in Topology 3 with DAB. $\mathrm{I}(\mathrm{Lm})$ - SMES current, Is - grid current after input filter, UCo - phase input voltage, U2T1 - secondary winding voltage of T1 transformer, Udc - dc link voltage, UCF1 - capacitor voltage CF1, I2T1, U2T1

- secondary winding current and voltage of transformer T1

of the rectifier and the voltage UCF1 of the chopper power supply capacitor.

Figures 13-16 show operation of an interface of power converter system. The topology consists of a three-level grid-connected active rectifier AC-DC and two bi-directional DC-DC converters, according to the topology presented in Fig. 6. Sinusoidal PWM modulation was used to control the three-level active bridge.
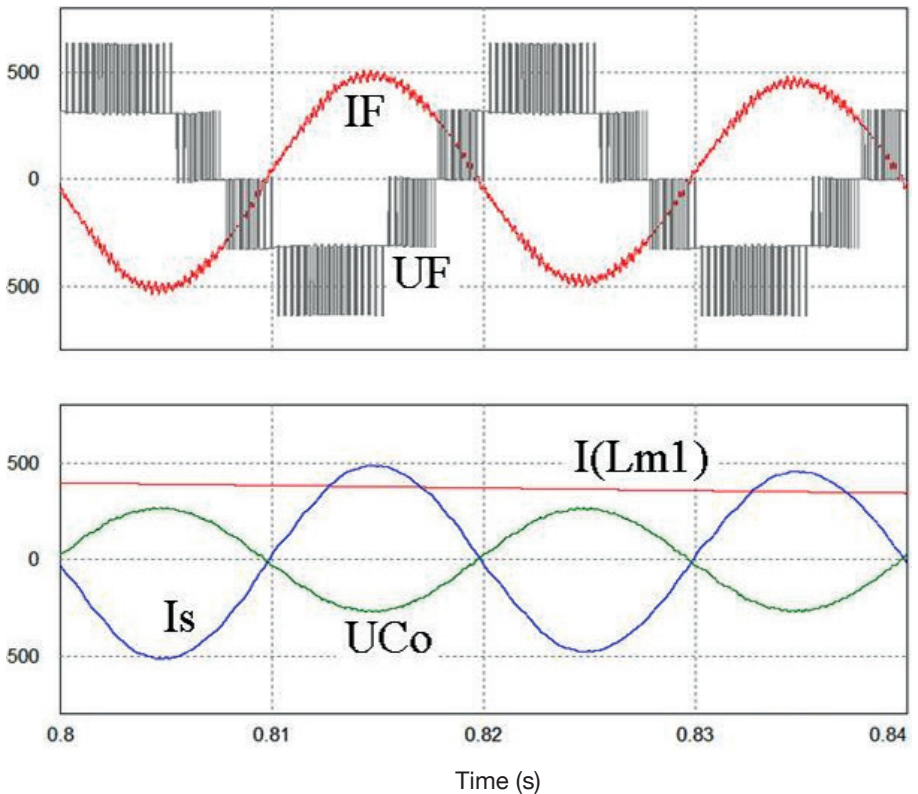

Fig. 14. Discharging process of SMES; energy is transferred back to the grid. UF - phase to phase input voltage without filter, IF - grid current without input filter, I(Lm) - SMES current, Is - grid current after input filter, UCo - phase input voltage 


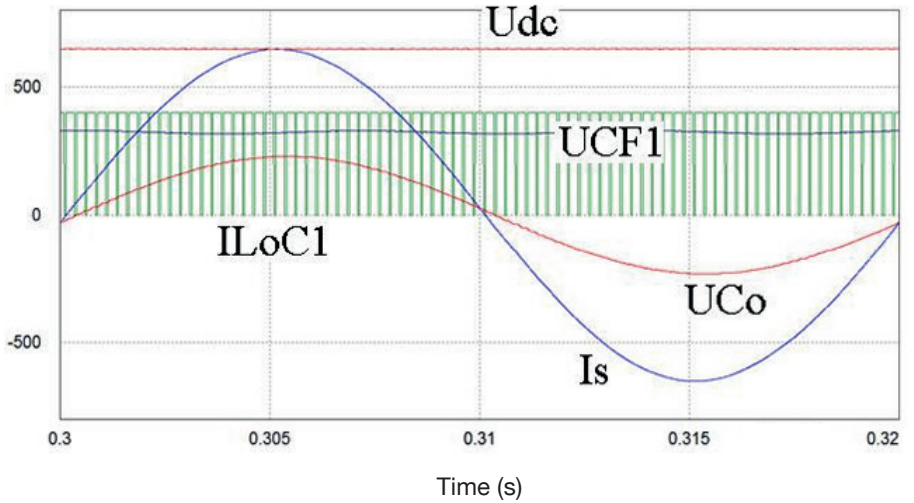

Fig. 15. Charging process of SMES in Topology 6. Is - grid current after input filter, UCo - phase input voltage. Udc - dc link voltage, UCF1 - capacitor voltage, ILoC1 - charging current of storage Lm1

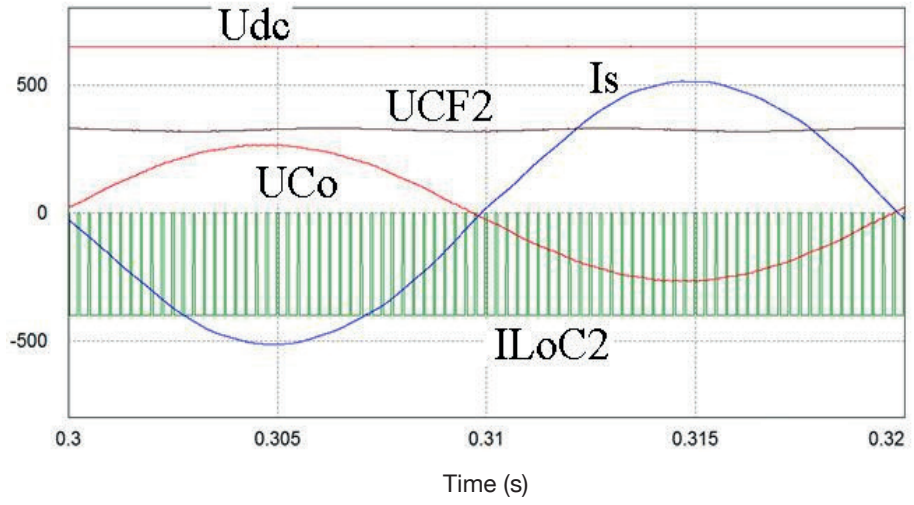

Fig. 16. Discharging process of SMES in Topology 6. Is - grid current after input filter, UCo - phase input voltage, Udc - DC-link voltage, UCF1 - capacitor voltage, ILoC1 - charging current of storage Lm1
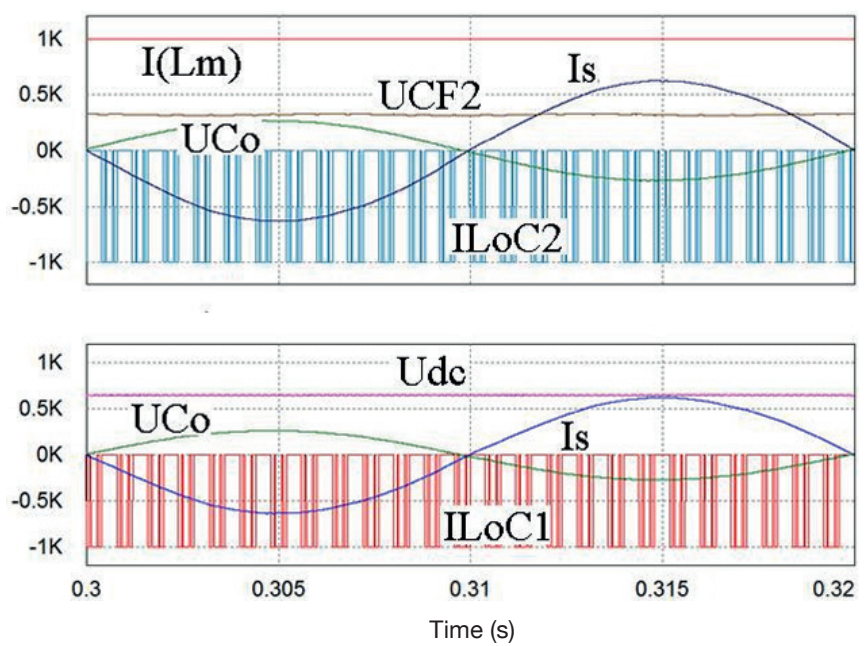

Fig. 18. Discharging process of SMES of Topology 7. Is - grid current after input filter, UCo - phase input voltage. Udc - DC-link voltage, UCF2 - capacitor voltage, ILoC2, ILoC1 - DC-DC converter currents during charging of energy storage $\mathrm{Lm}$

the grid voltage and current (UCo, Is) of the rectifier and DClink voltage (Udc) and voltage of the second section of DC circuit (UCF2).

Figures 17 and 18 show the operation of the interface of the power converter system during charging and discharging. The topology consists of an three-level grid-connected active AC-DC rectifier (see Fig. 7). The voltage in the DC-link is $\mathrm{Udc}=650 \mathrm{~V}$. The SMES current is ILm $=1000 \mathrm{~A}$. The effective value of charging and discharging current of energy storage $\mathrm{ILoC} 1=\mathrm{ILoC} 2$ is $580 \mathrm{~A}$ and average value $-350 \mathrm{~A}$. The active power determined by the current and phase voltage (Is, UCo) is $\mathrm{P}=240 \mathrm{~kW}$. The frequency of sinusoidal PWM in active rectifier is $\mathrm{f}_{\mathrm{pr}}=5 \mathrm{kHz}$ and the operating frequency of DC-DC converter is $\mathrm{f}_{\mathrm{cz}}=5 \mathrm{kHz}$.

Figure 17 shows currents of two DC-DC inverter sections (ILoC1 and ILoC2) when charging the energy storage with respect to the voltage and current of the rectifier and DC-link 
Table 2

Comparison of converter topologies for energy storage grid interfacing

\begin{tabular}{|c|c|c|c|c|c|c|}
\hline Topology No. & Grid transformer & AC-DC converter & DC-DC converter & DAB converter & Energy storage & Application power \\
\hline 1 & No & Two-level & Two-level & No & Single SMES & Low, middle \\
\hline 2 & Yes & Two-level & $3 \times$ two-level & No & Multiple SMES, SES & Middle \\
\hline 3 & No & Two-level & Two-level & Yes, two-level & Single SMES & Low, middle \\
\hline 4 & Yes & Two-level & $2 \times$ two-level & Yes, $2 \times$ two-level & Multiple SMES, SES & Middle, high \\
\hline 5 & Yes & Three-level & $2 \times$ two-level & Yes, $2 \times$ two-level & Multiple SMES & High \\
\hline 6 & Yes/No & Three- $n$-level & n x two-level & No & Multiple SMES & Middle, high \\
\hline 7 & Yes/No & Three- $n$-level & Three-level & No & Single SMES & Middle, high \\
\hline
\end{tabular}

voltage (Udc), and voltage of the second section of DC circuit (UCF2).

Figure 18 shows the same voltages and currents as in Fig. 17, but in the process of transferring energy from the SMES storage to the grid.

\section{Summary and conclusions}

The paper discusses some solutions of power electronics converters for coupling energy storages with grid power. Those systems are designed mainly for industrial plants, in which the energy is transmitted by internal networks with voltage level $3 \times 6 \mathrm{kV}$ and $3 \times 400 \mathrm{~V}$. In these conditions, energy storage must ensure the continuation of ongoing processes in the case of a sudden power failure and to reduce peak power resulting from the start-up of equipment and overloads. Additionally, these devices, due to the high-capacity energy storage, can collect energy during periods of her small consumption (tariffs) and giving back during the energy summit. The basic properties of the presented solutions are summarized as follows (Table 2).

The discussed grid interfaces relate to systems of low power supplied from the low voltage grid, and also supplied from the industrial plant $3 \times 6 \mathrm{kV}$ directly or by grid-transformers. We provide solutions to the two-level and three-level converters both AC-DC installable from the grid power supply and DC-DC converter connected directly to the storage.

The paper also presents solutions to the problem of cooperation with a single energy storage unit and with several storage units of the same electrical performance, and also with several units with different properties as supercapacitors (SES), low-temperature inductors (SMES) and batteries (accumulator). To meet the requirements of the cooperation of several types of storages with different electrical properties, it is suggested to use coupling via DAB circuit with high-frequency transformers.

Acknowledgements. This work was prepared in the framework of the PBS project, "Superconducting magnetic energy storage with a power electronic interface for the electric power systems", Contract no. PBS3/A4/13/2015.

\section{REFERENCES}

[1] Electricity Storage, Leading the Energy Transition Factbook, SBC Energy Institute, 2013.

[2] H. Majchrzak, "Energy storage as a remedy for RES problems", Czysta Energia 8/2014 [in Polish] (2014).

[3] Technology Roadmap IEA, Energy Storage, IEA, 2014 https://www.iea.org/publications/freepublications/publication/ TechnologyRoadmapEnergystorage.pdf

[4] J. Jensen and B. Sorensen, Fundamentals of Energy Storage, J. Wiley, New York, 1984.

[5] Y. Brunet, Energy Storage. Hoboken, Wiley, 2011.

[6] M.-C. Pera, D. Hissel, H. Gualous, and C. Turpin, Electrochemical Components, ISTE - J. Wiley, 2013.

[7] S.O. Amrouche, D. Rekioa, and T. Rekioa, "Overview of energy storage in renewable energy systems", 3rd International Renewable and Sustainable Energy Conference, 1-6 (2015).

[8] M. Bartosik, W. Kamrat, M.P. Kaźmierkowski, W. Lewandowski, M. Pawlik, T. Peryt, T. Skoczkowski, A. Strupczewski, and A. Szeląg, "Energy storage and hydrogen economy", Przegląd Elektrotechniczny 92 (12), 332-340 [in Polish] (2016).

[9] S. Vazquez, S. M. Lukic, E. Galvan, L.G. Franquelo, and J.M. Carrasco, "Energy storage systems for transport and grid applications" IEEE Trans. on Industrial Electronics 57(12), 3881-3895 (2010).

[10] X. Luo, J. Wang, M. Dooner, and J. Clarke, "Overview of current development in electrical energy storage technologies and the applied potential in power system operation," Appl. Energy 137, 511-536 (2015).

[11] G. Benysek, M.P. Kazmierkowski, J. Popczyk, and R. Strzelecki, "Power electronic systems as crucial part of smart grid infrastructure - a survey", Bull. Pol. Ac.: Tech. 59 (4), 445-473 (2011).

[12] M. Malinowski and M.P. Kaźmierkowski, "Simple direct power control of three-phase rectifier using space vector modulation - a comparative study", EPE European Power Electronics and Drives Journal, 13 (2), 28-33 (2003).

[13] H. Abu-Rub, M. Malinowski, and K. Al-Haddad, Power Electronics for Renewable Energy Systems, Transportation and Industrial Applications, IEEE Press and J. Wiley \& Sons Ltd., 2014. 
[14] P.J. Grabovic, P. Delarue, P. Le Moigne, and P. Bartholomeus, "A bidirectional three-level dc-dc converter for the ultracapacitor applications", IEEE Trans. Ind. Elect. 57(10), 3415-3430 (2010).

[15] S. Chakraborty, M.G. Simoes, and W.E. Kramer (Eds.), Power Electronics for Renewable and Distributed Energy Systems, Springer-Verlag London, 2013.

[16] M. Malinowski, M.P. Kaźmierkowski, and A.M. Trzynadlowski, "A comparative study of control techniques for PWM rectifiers in AC adjustable speed drives," IEEE Trans. Power Electron., 18(6), 1390-1396 (2003).

[17] F.A. Inthamoussou, J. Pegueroles-Queralt, and F.D. Bianchi, "Control of a supercapacitor energy storage system for microgrid applications", IEEE Trans. on Energy Conversion 28(3), 690-697 (2013)

[18] R. Barlik, M. Nowak, P. Grzejszczak, and M. Zdanowski, "Analytical description of power losses in a transformer operating in the dual active bridge converter", Bull. Pol. Ac. Tech. 64(3), 561-574 (2016).

[19] S.P. Engel, N. Soltau, H. Stagge, and R.W. De Doncker, "Dynamic and balanced control of three-phase high-power dualactive bridge dc-dc converters in dc-grid applications", IEEE Trans. Power Electron. 28(4), 1880-1889 (2013).

[20] W. Jing, Ch.H. Lai, S.H.W. Wong, and M.L.D. Wong, "Battery-supercapacitor hybrid energy storage system in standalone DC microgrids: a review", IET Renewable Power Generation, 11, 461-469, (2017).

[21] M. Momayyezan, D.B. W. Abeywardana, B. Hredzak, and V.G. Agelidis, "Integrated reconfigurable configuration for battery/ultracapacitor hybrid energy storage systems", IEEE Transactions on Energy Conversion 31(4) 1583-1590 (2016).

[22] H. Zhou, T. Bhattacharya, D. Tran, T.S.T. Siew, and A.M. Khambadkone, "Composite energy storage system involving battery and ultracapacitor with dynamic energy management in microgrid applications," IEEE Transactions on Power Electronics 26(3) 923-930 (2011).

[23] X. Zhao, Y.W. Li, H. Tian, and X. Wu, "Energy management strategy of multiple supercapacitors in a DC microgrid using adaptive virtual impedance", IEEE Journal of Emerging and Selected Topics in Power Electronics 4(4), 1174-1185 (2016).
[24] D.B. Wickramasinghe Abeywardana, B. Hredzak, V.G. Agelidis, and G.D. Demetriades, "Supercapacitor sizing method for energy-controlled filter-based hybrid energy storage systems", IEEE Trans. on Power Electronics, 32(2), 1626-1637 (2017).

[25] Zhe Zhang, Ziwei Ouyang, O.C. Thomsen, and M.A.E. Andersen, "Analysis and design of a bidirectional isolated DCDC converter for fuel cells and supercapacitors hybrid system", IEEE Trans. on Power Electronics 27(2), 848-859 (2012).

[26] S.D. Gamini Jayasinghe, D.M. Vilathgamuwa, and U.K. Madawala, "Diode-clamped three-level inverter-based battery/ supercapacitor direct integration scheme for renewable energy systems", IEEE Trans. on Power Electronics 26(12), 3720-3729 (2011).

[27] M. Nowak, J. Hildebrandt, and P. Łuniewski, "Converters with $\mathrm{AC}$ transformer intermediate link suitable as interfaces for supercapacitor energy storage", Proc. IEEE Power Electron. Spec. Conf. 5, 4067-4073 (2001).

[28] M.H. Ali, B. Wu, and R.A. Dougal, "An overview of SMES applications in power and energy systems," IEEE Trans. on Sustainable Energy 1(1) 38-47 (2010).

[29] J.X. Jin and X.Y. Chen, "Study on the SMES application solutions for smart grid", Physics Procedia 36, 902-907 (2012).

[30] P. McKenna, "Superconducting magnets for grid-scale storage", Technology Review, Energy, March 2011.

[31] P. Mukherjee and V.V. Rao, "Power system transient stability with SMES controlled by Artificial Intelligent Techniques", Electrical and Computer Engineering, 108-111 (2016).

[32] P.D. Baumaan, "Energy conservation and environmental benefits that may be realized from superconducting magneting energy storage", IEEE Trans. on Energy Conservation 7 (6) 253-259 (1992).

[33] W.K. Ham, S.W. Hwang, and J.H. Kim, "Active and reactive power control model of superconducting magnetic energy storage (SMES) for improvement of power system stability", $J$ Elect Tech 3(1), 1-7 (2008).

[34] Z. Wang, K.T. Chau, B. Yuwen, Z. Zhang, and F. Li, "Power compensation and power quality improvement based on multiple- channel current source converter fed HT SMES", IEEE Trans. on Applied Superconductivity 22(3), art. no. 5701204, (2013). 